when children are in a heightened positive mood. In previous research, framed safety messages have consisted of behaviourally targeted messages that emphasize avoiding risk behaviors leading to specific injuries and outcomes. The current study examined whether more general warning messages in framed contexts had a differential effect on reducing risk taking in children when in a heightened positive mood.

Methods/Approach 39 children (7-9 years) were exposed to a safety message (gain- or loss-frame) regarding play behaviors on an obstacle course. Children's risk-taking running the obstacle course was measured before and after a positive mood induction.

Results Participants who were exposed to loss-framed safety messages in both the general and behaviorally targeted groups demonstrated a significantly lower level of risk taking compared to baseline, whereas participants who were exposed to gain-framed safety messages in both groups performed at baseline levels. Regardless of whether children were exposed to general or behaviorally specific messages, gain and loss messaging counteracted the increase in risk taking when in a positive mood, but loss messages produced greater reductions in risk taking than gain messages.

Conclusion The results indicate that general messages can be as effective as behaviorally specific messages. Moreover, the loss-framed safety message had a greater effect on reducing risk-taking in children when in a heightened positive mood than the gain-framed safety message.

Significance and Contributions The results suggest that placing an emphasis on specific risk-taking behaviors and outcomes is not necessary in order to reduce risk-taking behaviours in school-aged children during play situations. This makes this intervention approach feasible to apply in situations in which there are a variety of potential risk behaviors which makes targeting a specific one likely to limit effectiveness of the intervention.

\section{NO HIT ZONES: AN INNOVATIVE APPROACH TO VIOLENCE PREVENTION}

${ }^{1}$ Julia Fleckman, ${ }^{1}$ Rachael Ruiz, ${ }^{2}$ Stacie Leblanc, ${ }^{1}$ Hannah Gilbert, ${ }^{1}$ Catherine Taylor. ${ }^{1}$ Tulane University School of Public Health and Tropical Medicine; ${ }^{2}$ The Up Institute

\subsection{6/injuryprev-2020-savir.116}

Statement of Purpose No Hit Zones (NHZs) are an emergent and highly promising public health strategy designed to change community-level social norms regarding the acceptability of physical punishment of children, an important approach for child physical abuse (CPA) prevention. NHZs are a multitiered strategic approach designed to: (1) promote an environment of safety such that no one will be hit, or witness hitting, within an organizational or community setting, and (2) reduce acceptance and use of PP within that setting, thereby ultimately reducing the incidence of CPA. This presentation will discuss the benefits and challenges of NHZ implementation, training resources available to implement an NHZ, and preliminary results from a NHZ evaluation at Children's Hospital of New Orleans.

Methods/Approach Training resources developed and results from a short-term evaluation of the NHZ intervention will be presented. Pre- and post-assessments were administered to all medical center staff invited to participate in the study $(n=507)$ before and after a mandatory NHZ training.
Measures were included to assess staff attitudes toward physical punishment, attitudes toward medical staff intervention when parents hit children, medical center policy and access to information on discipline, frequency of witnessing a parent hitting a child on the medical center property and whether or not they had intervened.

Results A pre-post analysis was completed via paired t-test, with significant results $(p<0.001)$ for a reduction in positive attitudes toward physical punishment, an increase in positive attitudes toward medical staff intervention when parents hit children, and willingness to intervene.

Conclusions The current results demonstrate promise for $\mathrm{NHZs}$ in reducing community-level risk for CPA.

Significance and Contributions to Violence and Injury Prevention Science NHZs are an innovative approach to family violence prevention at the community level. This presentation will discuss development of and preliminary results from the intervention evaluation.

\section{EMERGENCY MEDICAL RESPONSE TO US SPORTS INJURIES: PATIENTS, PLACES, AND PROCEDURES}

Viktor Bovbjerg. Oregon State University

10.1136/injuryprev-2020-savir.117

Statement of Purpose Emergency medical services (EMS) often respond to injuries sustained during sports. Characterizing such incidents can help schools/organizations and EMS agencies anticipate sports-related pre-hospital care needs, and coordinate their responses.

Methods/Approach We identified EMS runs associated with team sports (ICD-10 activity code Y93.6x), to a school, athletic field, or another athletic facility, using National Emergency Medical Services Information System 2017 data. We characterized incidents by patient characteristics, incident location, and pre-hospital medical procedures; we used ICD-10 diagnosis codes and SNOMED procedure codes.

Results Of 1107 EMS runs identified, the median patient age was 17 years (interquartile range 14-29), 29.5\% of patients were female, and $46.7 \%$ were white. The most common locations were athletic fields (517 incidents, 46.7\%) and schools (330, 29.8\%). Initial diagnoses were non-specific: the most common primary symptom was 'pain, unspecified' (34.7\%), and the most common primary impression was injury, unspecified' (53.7\%). Of 1656 procedures recorded, $287(17.3 \%)$ were conducted prior to EMS arrival. The most common preEMS procedure was splinting (32.4\% of pre-EMS procedures), and the most common EMS procedures were gaining intravenous access (33.7\%) and electrocardiographic monitoring (22.6\%).

Conclusions The vast majority of sports-related EMS responses were for teens and young adults, at known athletic venues, for musculoskeletal injuries and pain. Pre-EMS care often focused on stabilizing the injury, while EMS care often involved advanced assessment and pain relief/fluid administration. National EMS data, while informative, often lacked diagnostic detail, and the specific type of athletic facility; combining EMS data with hospital diagnoses, when available, would enable greater precision.

Significance and Contributions to Injury and Violence Prevention Science Characterizing sports-related EMS responses allows organizations with responsibility for athlete health and safety 